\title{
Theoretical Simulation of the Infrared Absorption Spectrum of the Strong Hydrogen and Deuterium Bond in 2-Pyridone Dimer
}

\author{
Noureddine Issaoui", Houcine Ghalla, Brahim Oujia \\ Quantum Physics Laboratory, Sciences Faculty of Monastir, Monastir, Tunisia \\ Email: issaoui_noureddine@yahoo.fr
}

Received August 1, 2012; revised September 2, 2012; accepted October 5, 2012

\begin{abstract}
This work presents a theoretical simulation of the infrared spectra of strong hydrogen bond in alpha-phase 2-pyridone dimers, as well as in their deuterium derivatives at room temperature. The theory takes into account an adiabatic anharmonic coupling between the high-frequency N-H(D) stretching and the low-frequency intermolecular N...O stretching modes by considering that the effective angular frequency of the fast mode $\mathrm{N}-\mathrm{H}(\mathrm{D})$ is assumed to be strongly dependent on the slow mode stretching coordinate N...O, the intrinsic anharmonicity of the low-frequency N...O mode through a Morse potential, Davydov coupling triggered by resonance exchange between the excited states of the fast modes of the two hydrogen bonds involved in the cyclic dimer, multiple Fermi resonances between the N-H(D) stretching and the overtone of the N-H(D) bending vibrations and the direct and indirect damping of the fast stretching modes of the hydrogen bonds and of the bending modes. The IR spectral density is computed within the linear response theory by Fourier transform of the autocorrelation function of the transition dipole moment operator of the N-H(D) bond. The theoretical line shapes of the $v_{\mathrm{N}-\mathrm{H}(\mathrm{D})}$ band of alpha-phase 2-pyridone dimers are compared to the experimental ones. The effect of deuteration is successfully reproduced.
\end{abstract}

Keywords: Strong Hydrogen Bound; Alpha-Phase 2-Pyridone Dimer; Infrared Spectral Density; Davydov Coupling; Morse Potential; Fermi Resonances

\section{Introduction}

The hydrogen bonding is responsible for the appearance of spectacular changes in IR spectra of associated molecules. This remark particularly concerns the $v_{\mathrm{X}-\mathrm{H}}$ proton stretching vibration bands, which are the attribute of the $\mathrm{X}-\mathrm{H}$ atomic groups in the $\mathrm{X}-\mathrm{H} \ldots \mathrm{Y}$ bridges. The main effects depend on a considerable low-frequency $v_{\mathrm{X}-\mathrm{H}}$ band shift, on the band integral intensity growth by even two orders of magnitude and on a noticeable increase of the band-width [1-3]. Numerous theoretical studies on hydrogen-bonded systems such as carboxylic acid have to explain the changes in the infrared spectrum induced by the formation of the H-bond Bridge.

In this work we present a theoretical approach for vibrational couplings in moderately strong hydrogenbonded systems and use it for simulating experimental infrared spectra for strong hydrogen-bonded of alpha solid-state phase 2-pyridone crystal dimers. It is shown alpha phase of 2-pyridone forms approximately a centrosymmetric dimer. In fact, that from the results given in

${ }^{*}$ Corresponding author. the original crystallographic work, it results that the dimers are not ideally centrosymmetric since one hydrogen bridge in a dimer is longer than the other. Without making significant errors, in the discussion of spectroscopic effects, one may assume that the dimers are approximately centrosymmetric. The alpha-phase crystals belong to the monoclinic system with $Z=8$ [4]. However, we think that for the IR spectroscopy reasons you may safely consider these dimers as centrosymmetric ones. Several studies show that 2-pyridone form centrosymmetric dimers $[5,6]$.

The studies have proved that in the case of cyclic dimers in this phase of 2-pyridone a single hydrogen bond is considerably stronger than a single hydrogen bond in a chain of molecules in the beta-phase. This fact suggests that the $\mathrm{N}^{+}-\mathrm{H} . . . \mathrm{O}^{-}$bonds exist only in the alpha-phase and that these bonds are obviously stronger than the N-H...O hydrogen bonds in the beta-phase, since ionic hydrogen bonds are generally known to be stronger than the neutral ones [7,8]. 2-pyridone is an important precursor of antibiotics, which are used as inhibitors of the DNA gyrase. Thus 2-pyridone [9] and its 
derivatives $[10,11]$ are widely investigated and exploited. Wuest and coworkers have proved that dipyridone can be formed by linking the two 2-pyridone using the functional groups like the acetylene or amine $[12,13]$. Asymmetric dipyridones are ready to form a dimer, whereas symmetric dipyridones are easy to self-assembly to form the linear and planar polymers in aprotic solvent [12]. The formation of the polymers via hydrogen bonding is an important approach of preparing liquid crystal and other functional polymers. There have been only a few reports on the hydrogen-bonding complexes formed by dipyridones. In addition, 2-pyridone dimer is an important reference system because it represents the rare case of a closed complex with two linear H-bonds. Recently, Wójcik presented a theoretical simulation of the bandshape and fine structure of $v_{\mathrm{N}-\mathrm{H}}$ stretching band for 2-pyridone- $\mathrm{H}$ and 2-pyridone-D [14], taking into account an adiabatic coupling between high-frequency N-H(D) stretching and low-frequency intermolecular N...O stretching modes, Davydov interactions and linear and quadratic distortions of potential energies for the low-frequency vibrations in the excited state of the N-H(D) stretching vibrations. The slow vibration modes were assumed as harmonic potentials. In other work, Wójcik [15] has presented a theoretical model for vibrational couplings in weak and moderately strong hydrogen-bonded systems and use it for modeling experimental infrared spectra for hydrogenbonded crystals and hexagonal ice. The model is based on vibronic-type couplings between high and low frequency modes in hydrogen bridges, Davydov interactions [16] and Fermi resonance $[17,18]$. It allows calculation of energy and intensity distributions in the infrared spectra of hydrogen-bonded systems. The present theory is based on strong anharmonic coupling between the highfrequency hydrogen stretching vibration $v_{\mathrm{N}-\mathrm{H}}$ and lowfrequency phonons, Davydov interactions and multiple Fermi resonances interaction between a fundamental vibration of a $v_{\mathrm{N}-\mathrm{H}}$ and one overtone or a combination tone of intermolecular vibration. Besides, this theory incorporated the intrinsic anharmonicity of the slow frequency mode through a Morse potential $[19,20]$ whereas the fast mode was considered as harmonic. Note that, the Morse potential is undoubtedly more realistic than the harmonic one to describe this slow frequency mode. The adiabatic approximation [21] have been performed for each separate part of the dimer together with a strong non-adiabatic correction via the resonant exchange between the excited states of the two fast mode moieties. Both quantum direct (relaxation of the high-frequency modes) and indirect (relaxation of the H-bond bridges) dampings of the systems $[22,23]$ were taken into account. This theory allows calculation of intensity distributions in the infrared spectra of hydrogen-bonded systems. The main pur- pose is to reproduce the experimental $v_{\mathrm{N}-\mathrm{H}}$ IR line shapes of hydrogen and deuterium bond in alpha-phase 2-pyridone crystal dimer at room temperature. We shall use infrared spectra of 2-pyridone in the alpha solid-state phase, measured by Flakus at the room temperature (Figures 1 and 2 from [24]).

The numerical results show that this theoretical approach allows fitting the experimental $v_{\mathrm{N}-\mathrm{H}}$ infrared line shapes of cyclic alpha-phase 2-pyridone crystal dimer and its deuterium derivative by using a minimum number of parameters. With such tools, experimentalist should be able to compare experimental and theoretical data in an easy-to-use way.

\section{Experimental Spectra}

It is of interested to note that, 2-pyridone compound which has been used in this investigation was a commercial substance (Sigma-Aldrich). The single crystals of the alpha phase formed rectangular plates. For the purpose of the experiment they were exposed by using on a tin diaphragm with a $1.5 \mathrm{~mm}$ hole diameter. It was used without further purification. Crystals of 2-pyridone suitable for spectral studies were obtained by crystallization from

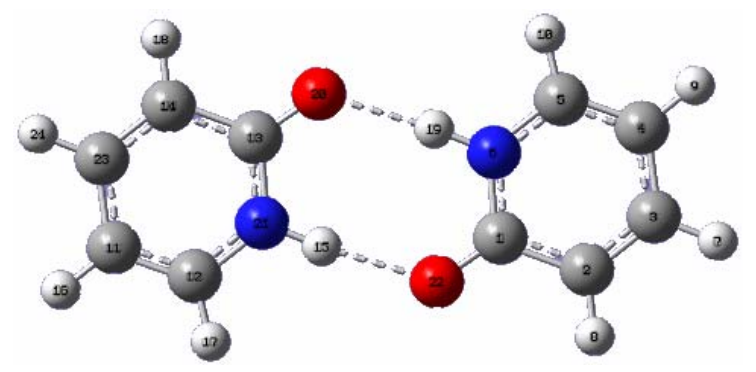

Figure 1. Geometry of the 2-pyridone dimer optimized at the HF/6-311 ${ }^{++}$G(d,p) level [26].

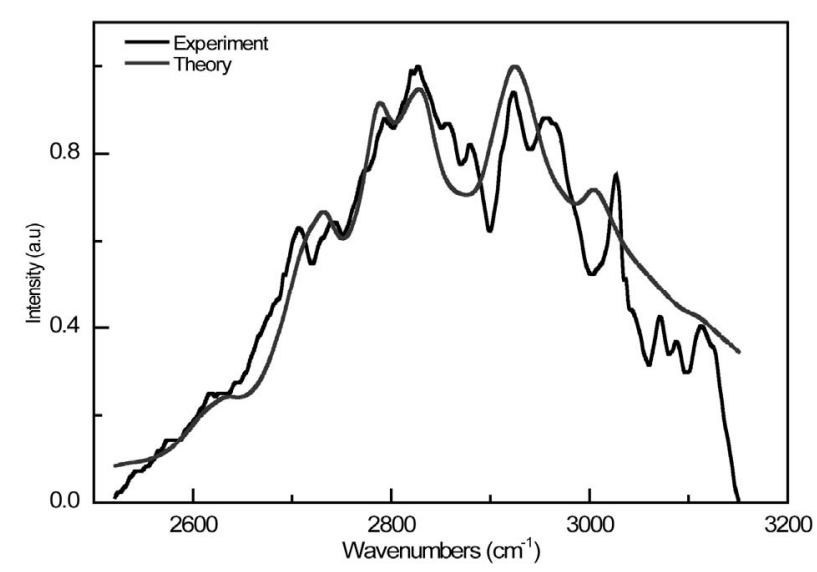

Figure 2. Comparison between the experimental and theoretical spectra of hydrogen-bonded in alpha-phase 2-pyridone at room temperature in the presence of three Fermi resonances. 
melt between two closely spaced $\mathrm{CaF} 2$ windows. The solid-state spectra were measured by a transmission method at room temperature with the help of the FT-IR Nicolet Magna 560 spectrometer using a non polarized beam. The spectra were measured at $2 \mathrm{~cm}^{-1}$ resolution. Measurements of spectra were completed in a similar way for the crystals of deuterium derivative of 2-pyridone, which was synthesized by evaporation of the solution in $\mathrm{D}_{2} \mathrm{O}$ under reduced pressure.

\section{Theory}

The present theory was developed in the framework of the adiabatic approximation [25]. The adiabatic approximation of the $\mathrm{N}-\mathrm{H}$ stretching vibrations leads to the description of each moiety by effective Hamiltonians of the H-bond Bridge: for a single $\mathrm{H}$-bond bridge, this effective Hamiltonian is either that of a harmonic oscillator, if the fast mode is in its ground state, or that of a driven harmonic oscillator if the fast mode is excited. When one of the two identical fast modes is excited, then, because of the symmetry of the cyclic dimer and because of coupling $\mathrm{V}_{0}$ between the two degenerate fast-mode excited states, an interaction occurs (Davydov coupling) leading to an exchange between the two identical excited parts of the dimer, as considered by Maréchal and Witkowski in their pioneering work [21]. Of course, this interaction between degenerate excited states is of non-adiabatic nature, although the adiabatic approximation was performed to separate the high- and low-frequency motions. Figure 1 present the geometry of the 2-pyridone dimer optimized at the HF/6-311 $1^{++} \mathrm{G}(\mathrm{d}, \mathrm{p})$ level [26], which is formed by two hydrogen bonds (lengths of N6-H19... $\mathrm{O} 20$ and $\mathrm{N} 21-\mathrm{H} 15 \ldots \mathrm{O} 22$ are $2.89559 \AA$ and $2.89558 \AA$, respectively).

Let $q_{1}$ and $q_{2}$, be the coordinates of the high-frequency $\mathrm{N}-\mathrm{H}$ stretching vibrations, in the first and second hydrogen bond, and $\mathrm{Q}_{1}$ and $\mathrm{Q}_{2}$ coordinates of the two low-frequency intermolecular N...O stretching modes. The two moieties of the system are exchanged by the symmetry C2 parity operator.

For the theory dealing with this system, the basic physical parameters are 1) vibration angular frequency $\omega^{\circ}$ of the two degenerates fast modes moieties when the $\mathrm{H}$ bond bridge is at the equilibrium, 2) vibration angular frequency $\Omega$ of the two degenerates H-bond bridge moieties, 3) dimensionless anharmonic coupling parameter $\alpha^{\circ}$ between the high frequency mode of one moiety and the H-bond bridge coordinate of the same moiety, 4) Davydov coupling $\mathrm{V}$ parameter between the degenerate first excited state of the two moieties high frequency mode, 5) direct and indirect damping parameters $\gamma$ and $\gamma, 6$ ) coupling parameters fi involved in the Fermi resonances coupling between the first harmonics of some bending modes of angular frequency and the first excited state of the $g$ symmetrized high frequency mode, 7) relaxations parameter of the first harmonic bending modes, and 8) absolute temperature $\mathrm{T}$.

\subsection{Full Hamiltonian of the System}

The evaluation of the spectral density of the hydrogen bond system requires the knowledge of the full Hamiltonian of the hydrogen bond system. For this purpose, it is important to describe the basic various vibration modes in the dimer. In our present work, for each part of the dimer, we have described the slow-frequency mode by a Morse potential, which can be written as follows:

$$
V_{\text {Morse }}=D_{e}\left[1-\mathrm{e}^{\left(-\beta_{e} Q \sqrt{\frac{M \Omega}{\hbar}}\right)}\right]^{2}
$$

$D_{e}$ is the dissociation energy of the Hydrogen Bond Bridge and $\beta_{e}$ is given by:

$$
\beta_{e}=\Omega \sqrt{\left(M / 2 D_{e}\right)} \sqrt{(\hbar / M \Omega)}
$$

The fast frequency-mode is considered to be harmonic. It is important to note that in the majority of recent works, the slow-frequency mode was assumed as harmonic [25].

Within the strong anharmonic coupling theory and the anharmonic approximation for the H-bond bridge, the corresponding Hamiltonians of the slow and high-frequency modes of the two moieties of the dimer are, respectively given, using dimensionless operators, by:

$$
\begin{gathered}
{\left[H_{\text {Slow }}\right]_{i}=(1 / 2) P_{i}^{2} \hbar \Omega+D_{e}\left[1-\mathrm{e}^{\left(-\beta_{e} Q_{i} \sqrt{M \Omega / \hbar}\right)}\right]^{2}} \\
{\left[H_{\text {Fast }}\right]_{i}=(1 / 2)\left(p_{i}^{2}+q_{i}^{2}\right) \hbar \omega\left(Q_{i}\right)}
\end{gathered}
$$

In these equations, $P_{i}$ are the dimensionless conjugate momenta of the H-bond bridges coordinates $Q_{i}$ of the two moieties, whereas $p_{i}$ and $q_{i}$ are the dimensionless coordinates and the conjugate momenta of the two degenerate high frequency modes of the two moieties. $\Omega$ is the angular frequency of the H-bond Bridge, whereas $\omega\left(Q_{i}\right)$ is that of the high frequency mode which is supposed to depend on the coordinate of the $\mathrm{H}$ bond bridge.

Using a Taylor development of the Morse potential, the Hamiltonian of the slow frequency modes given in Equation (3) can be rewritten as the sum of the Hamiltonian of a quantum harmonic oscillator and an anharmonic potential $V$ :

$$
\left[H_{\text {Slow }}\right]_{i}=\left[H_{\text {Slow }}\right]_{i}+V
$$

where $\left[H_{\text {Slow }}\right]_{i}$ and $V$ are respectively given by:

$$
\left[H_{\text {Slow }}\right]_{i}=(1 / 2)\left(P_{i}^{2}+Q_{i}^{2}\right) \hbar \Omega
$$




$$
V^{\circ \circ}=D_{e} \sum_{n=3}^{\infty}\left[\left((-1)^{n}\left(2^{n}-2\right)\right) / n !\right]\left(\beta_{e} Q_{i} \sqrt{M \Omega / \hbar}\right)^{n}
$$

Expansion to first-order of the angular frequency of the fast mode with respect to the coordinate of the $\mathrm{H}$-bond bridge leads to write:

$$
\omega\left(Q_{i}\right)=\omega^{\circ}+\alpha \Omega Q_{i}
$$

where $\omega^{\circ}$ is the angular frequency of the two degenerate fast modes when the corresponding H-bond Bridge coordinates are at equilibrium, whereas $\alpha$ is a dimensionless parameter which will appear to be an anharmonic coupling parameter.

In presence of damping, the thermal bath may be figured, by an infinite set of harmonic oscillators, and its coupling with the H-bond bridge are described by terms which are linear in the position coordinates of the bridge and of the bath oscillators:

$$
H_{\text {Damp }}=\sum(1 / 2)\left[\tilde{p}_{r}^{2}+\tilde{q}_{r}^{2}\right] \hbar \omega_{r}+\sum \tilde{p}_{r}\left[Q_{1}+Q_{2}\right] \hbar g_{r}
$$

Here, $\tilde{q}_{r}$ are the dimensionless position coordinate operators of the oscillators of the bath, $\tilde{p}_{r}$ are the corresponding conjugate moments, obeying the usual quantum commutation rules, $\omega_{r}$ are the corresponding angular frequencies and $g_{r}$ are the coupling between the H-bond bridges and the oscillators of the bath. Within the adiabatic approximation, the Hamiltonian of each moiety of the dimer takes the form of sum of effective Hamiltonians which are depending on the degree of excitation of the fast mode according to:

$$
\begin{aligned}
{\left[H_{\text {Adiab }}\right]_{i}=} & \sum\left[H^{\circ\{k\}}\right]_{i}\left|\{k\}_{i}\right\rangle\left\langle\{k\}_{i}\right| ; i=1,2 ; k=0,1 \\
{\left[H^{\circ\{k\}}\right]_{i}=} & (1 / 2) P_{i}^{2} \hbar \Omega+D_{e}\left[1-\exp \left(-\beta_{e} Q_{i} \sqrt{M \Omega / \hbar}\right)\right]^{2} \\
& +k \alpha^{\circ} Q_{i} \hbar \Omega+k \hbar \omega^{\circ}
\end{aligned}
$$

An excitation, of the fast mode of one moiety of the dimer is resonant with the excitation of the other moiety. Thus, a strong non-adiabatic correction [21] i.e. a Davydov coupling $V_{12}$, occurs between the two resonant states after excitation of one of the two fast modes, so that the full Hamiltonian of the two moieties is given by the equations:

$$
H_{\text {Tot }}=\left[H_{\text {Adiab }}\right]_{1}+\left[H_{\text {Adiab }}\right]_{2}+V_{12}+H_{\text {Damp }}
$$

with,

$$
V_{12}=\hbar V^{\circ}\left[\left|\{1\}_{1}\right\rangle\left\langle\{0\}_{2}|+|\{0\}_{1}\right\rangle\left\langle\{1\}_{2}\right|\right]
$$

\subsection{Autocorrelation Functions and Spectral Density}

The spectral density $I(\omega)$ of the $v_{\mathrm{N}-\mathrm{H}}$ mode is given, within the linear response theory [27,28], by the Fourier transform of the autocorrelation function $G(t)$ of transition moment operator of the fast mode :

$$
I(\omega)=2 \operatorname{Re} \int_{0}^{+\infty} G(t) \mathrm{e}^{-\mathrm{i} w t} \mathrm{~d} t
$$

Using the symmetry of the system, the ACF may be split into symmetric parts $(g)$ and antisymmetric parts (u).

In the presence of Davydov coupling, the autocorrelation function $(\mathrm{ACF})$ of the dipole moment operator of the fast mode may be written [25]:

$$
G(t) \propto[G(t)]_{g}\left[\left[G^{+}(t)\right]_{u}+\left[G^{-}(t)\right]_{u}\right] \mathrm{e}^{-\gamma^{\circ} t}
$$

Here, $\gamma$ is the natural width of the excited state of the high frequency mode, the expression of which has been calculated by Rösh and Ratner [22]. $[G(t)]_{g}$ is the reduced ACF of the $g$ part of the system related to Hamiltonian described the indirect damping. It's given by:

$$
\begin{aligned}
{[G(t)]_{g} } & \propto \mathrm{e}^{\mathrm{i} \omega^{\circ} t} \mathrm{e}^{-\mathrm{i} \frac{\alpha^{\circ}}{2} \Omega t} \mathrm{e}^{-\mathrm{i}\left[\frac{\beta}{\sqrt{2}}\right]^{2} \Omega t} \mathrm{e}^{-\mathrm{i}\left[\frac{\beta}{\sqrt{2}}\right]^{2}[\langle n\rangle+1 / 2]\left(2 \mathrm{e}^{-\gamma t / 2} \cos \Omega t-\mathrm{e}^{-\gamma t}\right)} \\
& \times \mathrm{e}^{\mathrm{i}|\beta|^{2} \mathrm{e}^{(-\gamma t / 2)} \sin \Omega t}
\end{aligned}
$$

In these equation, $<n>$ and $\beta$ are respectively the thermal average of the occupation number of the quantum harmonic oscillator describing the $\mathrm{H}$-bond bridge and the effective dimensionless anharmonic coupling parameter related to $\alpha^{\circ}$. These terms are given by:

$$
\begin{gathered}
\beta=\alpha \frac{\left[4 \Omega^{2}+\gamma^{2} \Omega^{2}\right]^{1 / 2}}{2 \sqrt{2}\left[\Omega^{2}+\left(\gamma^{2} / 4\right)\right]} \\
\langle n\rangle=1 /\left(\mathrm{e}^{(\hbar \Omega / k T)}-1\right)
\end{gathered}
$$

Note that by "reduced", we mean that a partial trace has to be performed over the thermal bath because of the coupling between the symmetric Hamiltonian and the surrounding. In the last equation, $\left[G^{+}(t)\right]_{u}$ and $\left[G^{-}(t)\right]_{u}$ are the two $(u)$ ACFs which are affected only by the Davydov coupling. They are given by [29]:

$$
\begin{aligned}
{\left[G^{ \pm}(t)\right]_{u} } & \propto \sum_{n} \sum_{n_{\mu}} \mathrm{e}^{-(n \hbar \Omega / k T)}\left[1 \pm(-1)^{n_{\mu}+1}\right]^{2} \\
& \times\left|C_{n_{\mu}, \mu}^{ \pm}\right|^{2} \mathrm{e}^{\mathrm{i} \omega_{\mu}^{ \pm} t} \mathrm{e}^{-\mathrm{i} n_{\mu} \Omega t}
\end{aligned}
$$

where, $C_{n_{\mu}, \mu}^{ \pm}$are the expansion coefficients of the eigenvectors on the basis of the eigenstates of the Hamiltonian of the quantum harmonic oscillator. One may observe that the angular frequency $\omega^{\circ}$ of the high frequency mode must be decreased by a factor $1 / \sqrt{2}$ on $D$ isotopic substitution of the proton involved in the H-bonds. Besides, according to Maréchal and Witkowski theory 
[21], the anharmonic coupling parameter $\alpha^{\circ}$ must also be reduced by this factor upon this same substitution, whereas the frequency $\Omega$ of the H-bond bridge has no reason to be modified.

Following Equations (15) and (19), the spectral density of the dimer involving Davydov effect can be written as:

$$
\begin{aligned}
I(\omega) \propto 2 \operatorname{Re} \int_{0}^{+\infty}[G(t)]_{g} & {\left[\left[G^{+}(t)\right]_{u}+\left[G^{-}(t)\right]_{u}\right] } \\
& \times \mathrm{e}^{-\gamma^{\circ} t} \mathrm{e}^{-\mathrm{i} w t} \mathrm{~d} t
\end{aligned}
$$

That may be written formally:

$$
I(\omega) \propto I^{+}(\omega)+I^{-}(\omega)
$$

with respectively:

$$
I^{ \pm}(\omega) \propto \sum_{m} \sum_{n}\left\{P_{m, n}\right\} \sum_{n_{\mu}} \sum_{n}\left\{A_{m, n}^{n_{\mu}, \mu}\right\}
$$

In view of the above equations, the components appearing here are given by the following equations [29]:

$$
\begin{gathered}
\left\{A_{m, n}^{n_{\mu}, \mu}\right\}=\mathrm{e}^{\lambda \bar{n}_{\mu}}\left[1 \pm(-1)^{n_{\mu}+1}\right]^{2} \times\left|C_{n_{\mu}, \mu}^{ \pm}\right|^{2}\left\{I_{m n_{\mu}, \mu}^{ \pm}(\omega)\right\} \\
\left\{I_{m n n_{\mu}, \mu}^{ \pm}(\omega)\right\} \propto\left(\gamma_{m, n}\right) /\left\{\left(\omega-\Omega_{m n_{\mu}, \mu}^{ \pm}\right)^{2}+\left(\gamma_{m, n}\right)^{2}\right\}, \\
\Omega_{m n_{\mu}, \mu}^{ \pm}=\omega^{\circ}-\left[\left(m-n+n_{u}\right) \Omega-\omega_{u}^{ \pm}\right]-\alpha \sqrt{2} \Omega, \\
\gamma_{m, n}=(m+n) \gamma+\gamma^{\circ}, \\
P_{m, n}=\left\{[1+\langle n\rangle]^{m}\langle n\rangle^{n}|\beta / \sqrt{2}|^{2(m+n)}\right\} / m ! n !
\end{gathered}
$$

Recall that $\langle n\rangle$ is the thermal average of the number occupation operator of the H-bond bridge vibration given by Equation (18), which is a function of the angular frequency $\Omega$ of the H-bond bridge and of the absolute temperature T. Besides, $\beta$ is the effective anharmonic coupling parameter given by Equation (17), which is a function of the anharmonic coupling parameter $\alpha^{\circ}$ between the slow and fast mode, and of the angular frequency $\Omega$ and the damping parameter $\gamma$ of the $\mathrm{H}$-bond bridge.

\subsection{Situation with Fermi Resonances}

The previous treatment was developed with the neglect of Fermi resonances. Now, suppose the situation where this later effect is taken into account. It is resulting from the interactions occurring between the first excited state of the high frequency mode and the first harmonic of some bending modes. As it was stated by Maréchal and Witkowski [21], if Fermi resonances are taken into account, one has to consider one fast mode, one slow mode and one bending mode for each hydrogen bond of the cyclic dimer. If we take into account Fermi resonances
[29], they affect only the g state of the system. As a consequence, the autocorrelation functions $\left[G^{-}(t)\right]_{u}$ are not modified. In the presence of Davydov coupling and Fermi resonances, the ACF can be written following [29]:

$$
[G(t)]_{g} \propto \sum_{n} \sum_{\mu} \mathrm{e}^{-\lambda m}\left|a_{\{\mu, 0, m\}_{g}}\right|^{2}\left[\mathrm{e}^{\mathrm{i}\left\{\omega_{\mu}\right\}_{g} t / \hbar}\right] e^{-\mathrm{i} m \Omega t}
$$

Here, $\left\{\omega_{\mu}\right\}_{g}$ are the eigenvalues appearing in Equation (29) whereas, $a_{\{\mu, 0, m\} g}$ are the expansion coefficients defined by Equation (30).

$$
\left[H_{D}^{F}\right]_{g}\left|\varphi_{\mu}\right\rangle_{g}=\hbar\left|\varphi_{\mu}\right\rangle_{g}\left\{\omega_{\mu}\right\}_{g}
$$

With

$$
\left|\varphi_{\mu}\right\rangle_{g}=\sum_{m} \sum_{g}\left\{a_{\mu, l, m}\right\}\left|\psi_{l, m}\right\rangle_{g}
$$

The $g$ states involved in the above expansions are defined by:

$$
\begin{aligned}
& \left|\left\{\Psi_{0, m}\right\}_{g}\right\rangle=\left|\{1\}_{g}\right\rangle\left|\left\{0_{1}\right\}_{g}\right\rangle\left|\left[0_{2}\right]_{g}\right\rangle\left|(m)_{g}\right\rangle \\
& \left|\left\{\Psi_{1, m}\right\}_{g}\right\rangle=\left|\{0\}_{g}\right\rangle\left|\left\{2_{1}\right\}_{g}\right\rangle\left|\left[0_{2}\right]_{g}\right\rangle\left|(m)_{g}\right\rangle \\
& \left|\left\{\Psi_{2, m}\right\}_{g}\right\rangle=\left|\{0\}_{g}\right\rangle\left|\left\{0_{1}\right\}_{g}\right\rangle\left|\left[2_{2}\right]_{g}\right\rangle\left|(m)_{g}\right\rangle
\end{aligned}
$$

Here, the kets

$$
\left|\{k\}_{g}\right\rangle,\left|(m)_{g}\right\rangle \text { and }\left|[1]_{\mathrm{g}}\right\rangle
$$

are the $g$ eigenstates of respectively the symmetrized high frequency quantum harmonic oscillator, the slow frequency quantum harmonic oscillator and the bending frequency quantum harmonic oscillator. The Fermi resonance mechanism, characterized by the coupling parameter $f_{i}$, is described by the following coupling operators $\hbar f$ which express the non-resonant exchanges between the state $\left|\{1\}_{j}\right\rangle$ of the $j$ th fast mode and second damped excited state $\left|[2]_{j}\right\rangle$ of the $j$ th bending mode. The introduction of the Fermi resonance coupling effects in the lineshape is presented by the complexes angular frequency gap $\Delta_{i}$ :

$$
\Delta_{i}=\Delta_{i}^{\circ}-i \gamma_{i}^{\delta}
$$

with

$$
\Delta_{i}^{\circ}=-\omega^{\circ}-2 \omega_{i}^{\delta}
$$

where $\omega_{i}^{\delta}$ is the frequency of the bending modes $\left(i=1,2, \cdots, n_{F}\right)$.. The imaginary part in this gap is related to the lifetime of the corresponding excited states. Recall that the Hamiltonian of the dimer involving Davydov coupling, Fermi resonances between the $g$ excited state of the fast mode and the $g$ first harmonics of the bending mode, together with the damping of theses excited states is: 


$$
\left[H_{\text {Fermi+Dav }}\right]_{g}=\left(\begin{array}{cccccc}
{\left[H^{\{1\}}\right]} & \hbar f_{1} & \hbar f_{2} & \hbar f_{3} & \cdots & \hbar f_{n F} \\
\hbar f_{1} & H^{\{0\}}+\hbar \Delta_{1} & & & & \\
\hbar f_{2} & & H^{\{0\}}+\hbar \Delta_{2} & & & \\
\hbar f_{3} & & & H^{\{0\}}+\hbar \Delta_{3} & \\
\cdots & & & & \cdots & \\
\hbar f_{n F} & & & & & H^{\{0\}}+\hbar \Delta_{n F}
\end{array}\right)
$$

with

$$
H^{\{0\}}=\left[a^{+} a+\frac{1}{2}\right] \hbar \Omega
$$

and

$$
\begin{aligned}
{\left[H^{\{1\}}\right]_{g}=} & {\left[\left(a^{+} a+\frac{1}{2}\right)+\alpha\left(a^{+}+a\right)\right] \hbar\left(\Omega-\mathrm{i} \frac{\gamma}{2}\right) } \\
& +\hbar \omega^{\circ}-\frac{\alpha}{2} \hbar \Omega
\end{aligned}
$$

In these two last equations, $\mathrm{a}^{+}$and a are the boson operators obeying $\left[a^{+}, a\right]=1$ and $i^{2}=-1$.

\section{Results and Discussions}

In our theoretical approach, we have assumed that the 2-pyridone dimer crystal in alpha-phase is of approximately centrosymmetric type. In the case of the interprettation of the spectra of alpha-phase 2-pyridone crystal, this interpretation cannot be relied on since the N-H...O bonds are much shorter than the sulfur atoms containing hydrogen bonds. Most probably, the purely vibrational approach to solving the problem of the exciton interacttions in the hydrogen bond dimers should be abandoned. However, the contribution of electronic interactions to the vibrational exciton interactions between the hydrogen bonds in the dimers, expressed by the electronic coordinates, should be taken account.

From these considerations some consequences result for the electronic structure of 2-pyridone dimers in alpha-phase crystals. This is most probably the structure of a larger contribution of the zwitterion electronic structure. Such a continuous structure of the $\pi$-electron cloud in the pyridine rings, in cyclic $\mathrm{N}^{+}-\mathrm{H} . . . \mathrm{O}^{-}$hydrogen bond dimers allows for an effective ("head-to-tail") coupling of the hydrogen bonds in the cyclic dimers. The experimental and theoretical results allow us to state that, for alpha-phase 2-pyridone studied the $v_{\mathrm{N}-\mathrm{H}}$ absorption bands of their dimers observed in the IR spectra are similar in structure and are very broad. This means that the mechanism of formation of these bands should, first of all, involve the participation of the cyclic structure, responsible for the formation of the hydrogen bond. Insignificant changes in the shape of the dimers and their deuterated derivatives bands observed in alpha-phase 2-pyridone. We believe that the interaction of the two intermolecular bonds $\mathrm{N}-\mathrm{H} . . . \mathrm{O}$ in the cyclic complex plays a determining role in the formation of the broad absorption band.

The main object in this work is to reproduce theoretically the IR spectra of alpha-phase 2-pyridone at $300 \mathrm{~K}$. In a first place we have reproduced the $v_{\mathrm{N}-\mathrm{H}(\mathrm{D})} \mathrm{IR}$ spectra of alpha-phase 2-pyridone dimer crystal by comparing theoretical line shapes. The spectral densities are computed by Equation (19) after construct and diagonalize the full Hamiltonian in a truncated basis. The stability of the computed spectra with respect to the size of this basis set was carefully checked. The stability of the spectra was also checked versus the order of the Taylor expansion of the Morse potential. Thus, in the first place, we have considered the general physical situation in which multiple Fermi resonances are taken into account and for which the spectral density is given by Equations (19), (20) and (28). The procedure we have used is the fitting of the experimental line shapes by optimizing the values of the basic parameters.

Figures 2 and 3 present the theoretical spectra of alpha-phase 2-pyridone dimer crystal and its deuterated derivative where multiple Fermi resonances have been introduced in our theoretical approach. We have performed numerical experimentation by increasing progressively the number of Fermi resonances. The spectral densities, presented in this Figure, are computed in the presence of three Fermi resonances. Tables 1 and $\mathbf{2}$ show the parameters involved in the calculations.

The examination of the spectra obtained using our theoretical model shows that there is a good agreement between theory and experiment. It is important to emphasize that if Fermi resonances are acting, one observes the classical behavior of the Fermi resonance, which is well described in the literature: the displacement of the bands and the redistribution of the intensities in the bands. However, only the intensities at high frequencies are redistributed if one increases the number of Fermi resonances. In fact, a recent study on acetic acid dimers in the liquid phase [30] did not show any wavelength dependence of their dynamics.

Figures $\mathbf{4}$ and $\mathbf{5}$ show the theoretical line shapes of alpha-phase 2-pyridone dimer crystal and its deuterated 
derivative calculated by Equations (16), (19) and (20) when Fermi resonances are ignored. In both situations, the theoretical line shapes appear as red lines whereas the experimental ones are black lines.

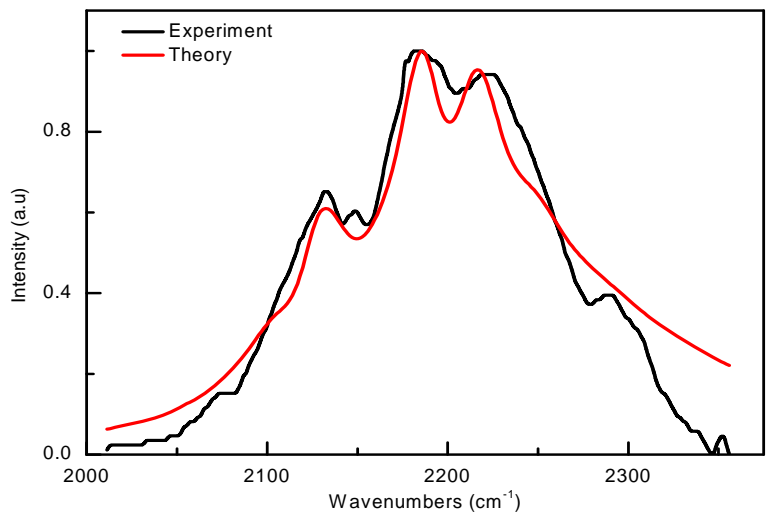

Figure 3. Comparison between the experimental and theoretical spectra of deuterium-bonded in alpha-phase 2-pyridone at room temperature in the presence of three Fermi resonances.

Table 1. Parameters used for fitting the experimental spectra of alpha-phase 2-pyridone at room temperature in the presence of three Fermi resonances.

\begin{tabular}{cccccccc}
\hline Species & $\omega^{\circ}\left(\mathrm{cm}^{-1}\right)$ & $\Omega\left(\mathrm{cm}^{-1}\right)$ & $\alpha^{\circ}$ & $\mathrm{V}^{\circ}$ & $\gamma^{\circ}\left(\mathrm{cm}^{-1}\right)$ & $\gamma\left(\mathrm{cm}^{-1}\right)$ & $\eta$ \\
\hline O-H & 2920 & 100 & 0.69 & -0.48 & 0.16 & $0.1 . \Omega$ & 0.9 \\
O-D & 2151 & 80 & 0.20 & -0.44 & 0.11 & $0.1 . \Omega$ & 0.5 \\
\hline
\end{tabular}

Table 2. Fermi coupling parameters (in $\mathrm{cm}^{-1}$ ) used for fitting experimental spectra of phase 2-pyridone at room temperature.

\begin{tabular}{cccccccccc}
\hline Species & $\boldsymbol{f}_{1}$ & $\boldsymbol{f}_{\mathbf{2}}$ & $\boldsymbol{f}_{\boldsymbol{3}}$ & $\Delta_{\mathbf{1}}$ & $\Delta_{\mathbf{2}}$ & $\Delta_{3}$ & $\gamma_{\boldsymbol{\delta} \mathbf{1}}$ & $\gamma_{\delta \mathbf{2}}$ & $\gamma_{\delta 3}$ \\
\hline $\mathbf{H}$ & -80 & 90 & 100 & 10 & 10 & 10 & 0.1 & 0.1 & 0.1 \\
$\mathbf{D}$ & -80 & 90 & 125 & 15 & 10 & 15 & 0.2 & 0.2 & 0.2 \\
\hline
\end{tabular}

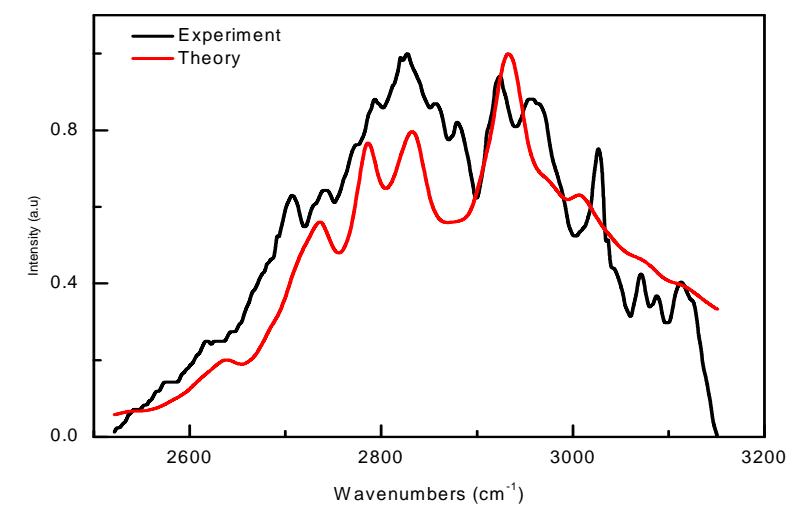

Figure 4. Comparison between the experimental and theoretical spectra of hydrogen-bonded in alpha-phase 2-pyridone at room temperature when the Fermi resonances are ignored.

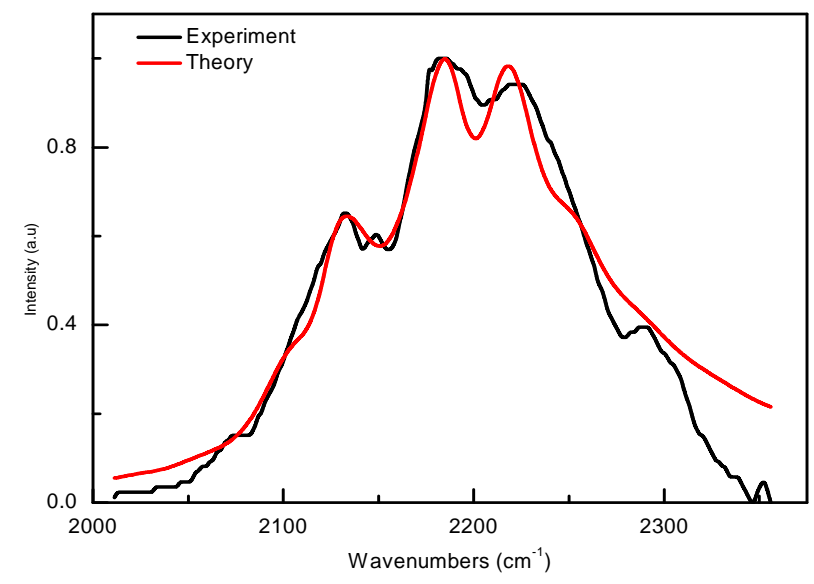

Figure 5. Comparison between the experimental and theoretical spectra of deuterium-bonded in alpha-phase 2-pyridone at room temperature in the absence of Fermi resonances.

In order to improve the validity of the present model, let us comment the magnitude of the parameters that we have used. Both the anharmonic coupling parameter and the angular frequencies of the high frequency mode for alpha-phase 2-pyridone in all treated situations, their magnitudes is decreased by a factor which is different from $\sqrt{2}$. This result is in agreement with theory when passing from the $\mathrm{H}$ to the $\mathrm{D}$ species and when the slow mode is assumed to be of Morse type (anharmonic potential). Indeed, according to [30,31], the isotope ratio

$$
\chi=\frac{\langle\omega\rangle_{H}}{\langle\omega\rangle_{D}}
$$

for hydrogen bonds $\mathrm{O}-\mathrm{H} . . . \mathrm{O}$ depends on the bond strength, it is close to the harmonic value $\sqrt{2}$ for weak bonds and decreases with the bond strength reaching minimum near $\chi=0.9$ for low-barrier bonds. In our cases, to obtain good agreement between the theoretical and experimental one, we have used:

$$
\frac{\alpha_{H}^{\circ}}{\alpha_{D}^{\circ}}=3.415, \frac{\left\langle\omega_{H}\right\rangle}{\left\langle\omega_{D}\right\rangle}=1.306 \text { and } \frac{V_{H}}{V_{D}}=1.118
$$

It is important to note that the introduction modulation of the equilibrium positions of the fast modes $\left(q_{e}\right)$ and the quadratic dependence of their frequencies on $Q_{i}$ which can represented by two expansions to the second order:

$\omega\left(Q_{i}\right)=\omega^{\circ}+\alpha Q_{i}+\beta Q_{i}^{2} ; q_{e}\left(Q_{i}\right)=q_{e}{ }^{\circ}+f^{\circ} Q_{i}+g^{\circ} Q_{i}^{2}$

Recall that in present work and following Maréchal and Witkowski [21], we have only used the first order dependence of the angular frequency of the fast modes on the coordinate of the slow modes $\left(Q_{i}\right)$ (see Equation (40) and we have neglected the modulation of the equilibrium positions of the fast modes and the quadratic dependence of their frequencies on $\left(Q_{i}\right)$ [32] as conformed 
by several experimental correlations [33,34]. We have shown in recent [32] work that the fine structure of the IR $v_{\mathrm{X}-\mathrm{H}}$ stretching band are connected with the new anharmonic coupling parameters (i.e. $\beta, f^{\circ}$ and $g^{\circ}$ ), whereas these parameters do not manifest markedly in effects of temperature and deuteration on the IR spectra. Besides, the account for all these parameters does not affect the similarity of the spectra in gas and condensed phases [35].

The isotope ratio $\chi$ of centers of gravity of band of light and deuterated alpha-phase 2-pyridone dimer is 1.306 . The values obtained here are also in satisfactory agreement with the experimental data reported by Odinokov, et al. [36] for complexes of carboxylic acids with various bases and are comparable to those found in our recent work dealing with the H/D isotopic effects in H-bond spectra [33]. However, these ratios are different from those used by Blaise, et al. [37] since in their approaches dealing with theoretical interpretation of the IR line shapes of liquid and gaseous acetic acid [38] and gaseous propynoic and acrylic acid dimers [29], low and high-frequency hydrogen stretching vibrations in individual hydrogen bonds are assumed to be harmonic whereas in the present work we use a Morse potential in order to describe the anharmonicity of the H-bond Bridge. Recall that the removal of the harmonic approximation for the slow modes by introducing Morse potential in place of harmonic one has been done by Leviel and Maréchal [39] in a model similar to the present one, involving cyclic dimer, however without damping. They have shown that the value of the angular frequency of the slow mode which must be used to the experimental lineshape is more close to the experimental value when the anharmonicity of the bridge is introduced.

Now let us look to the strongest hydrogen bonds observed in alpha-phase 2-pyridone dimers. Generally this type of hydrogen bonds is observed in ion molecular complexes of the (AHA) ${ }^{-}$and $(\mathrm{BHB})^{+}$types $[40,41]$; however, it is especially difficult to study the spectral properties of charged complexes under conditions of weak interactions with the medium. In the present approach, we take into account the natural width of the excited states of the fast mode due to the medium (direct relaxation) and the damping of the H-bond Bridge (indirect relaxation). The direct relaxation is included following quantum treatment of Rösch and Ratner [22] whereas the indirect one was taken into account via the approach of Louisell and Walker [42] dealing with the relaxation of driven damped quantum oscillators studied initially by Feynman and Vernon [43] and later by Louisell [44]. The values of the direct and indirect relaxation parameters reflecting the effect of the medium used presently for alpha-phase 2-pyridone are of the same magnitude as those used by Blaise, et al. $[29,37,38]$ in their study deal- ing with acetic acid in the gas phase, whereas the indirect damping at $298 \mathrm{~K}$ for acetic acid in crystalline state is $\gamma=$ $1 \mathrm{~cm}^{-1}$. One may ask whether the indirect damping used for crystalline state is weaker than that used for the gaseous phase since the indirect relaxation is ought to be larger in the solid state. The reason is that in the gas phase the indirect damping is an effective one which is the result of the combination of the indirect damping and of the rotational structure [45]. In neutral systems, the strongest hydrogen bonds of the N-H...O type are formed in self-associates of alpha-phase 2-pyridone and, seemingly, phosphinic acids [46], and it is only due to the high thermal stability of these associates that one is able to observe their IR spectra in the gas phase, at which cyclic dimers are in equilibrium with monomeric molecules $[47,48]$. This made it possible to analyze the dependence of the bandshape of the $v_{\mathrm{N}-\mathrm{H}}$ band, which serves as an important criterion in the choice of theoretical models. The parameters of this broad band and its shape turned out to be almost independent of the bonding frequency.

In order to obtain a good agreement with the experimental line shapes, we have take into account some breaking of the IR selection rule for the centrosymmetric cyclic dimer, via a large amount $(\eta=0.5 ; 0.9)$ of forbidden Ag transition. Recall that in general way the quality of the fitting is weakly improved by taking small values for $\eta$ which lying between 0 and 1 . This assumption was initially introduced by Flakus [49]. This is a general trend which has been observed recently in the cases of the centrosymmetric cyclic dimers of gaseous acetic acid [37]. Note that, according to Flakus hypothesis, the lack of forbidden transition ought to be stronger in the solid state (value near $\eta=1$ ) than in the gaseous one and we must keep in mind that the Flakus assumption has been seen by this author to be unavoidable in diverse crystalline H-bonded carboxylic acids such as for instance glutaric [50] and cinnamic [51] acids and particularly in centrosymmetric $\mathrm{H}$-bonded dimers.

Figure 6 presents the theoretical spectra of alphaphase 2-pyridone dimer where multiple Fermi resonances have been introduced. The number of Fermi resonances $n_{F}$ is increasing from zero (without Fermi resonances) to $3\left(n_{F}=0,1,2\right.$ and 3$)$. The numerical results show that Fermi resonances appear to play an important role especially for the hydrogenated species whereas the Fermi resonances effect does not affect markedly the N-D derivative species. It may be explain by the fact that Fermi resonances mechanism may involve the N-H bond bending-in-plane vibrations in their first overtone states. So the coupling between the electronic systems with the electrons of the associated carboxyl groups implies a stabilization of the dimers. On the other hand, each deformation of the dimers provides a destruction of this 


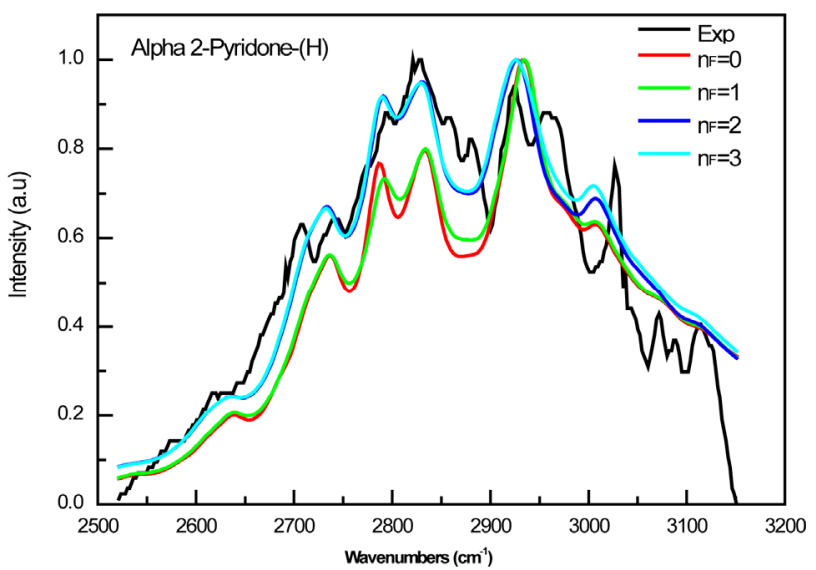

(a)

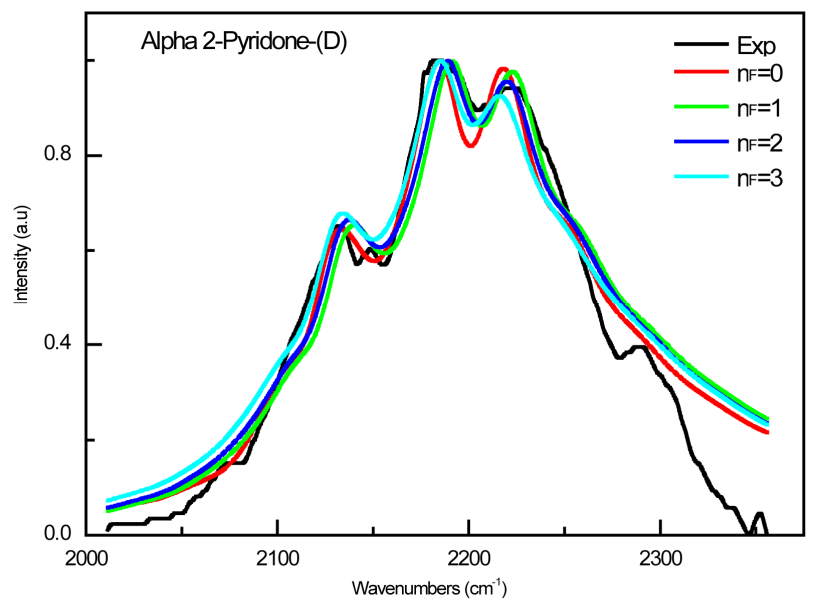

(b)

Figure 6. Multiple Fermi resonance effect on the IR spectral densities of hydrogen (a) and deuterium (b) bonded in alpha-phase 2-pyridone. The number of Fermi resonances $\mathrm{nF}$ is increasing from zero (without Fermi resonances) to 3.

stabilization mechanism. This is the most probable source of the anharmonic coupling involving the proton stretching and the proton bending-in-plane vibrations in the dimer of carboxylic acids. We think that the sensitive improvement of the theoretical line shape of strongly bound dimers of alpha-phase 2-pyridone by introduction of Fermi resonances might be due to some whole effect in which the Fermi resonances assisted by the strong anharmonic coupling would be augmented by the combination of the Davydov coupling and of the quantum direct and indirect damping. It appears from the present theoretical study that the simple model of Davydov coupling taking into account especially Fermi Resonances and quantum direct and indirect damping, is able to reproduce the experimental line shapes. When one of the two identical fast modes is excited, then, because of the symmetry of the cyclic dimer, and of Davydov coupling $\mathrm{V}_{\mathrm{D}}$ between the two degenerate fast mode excited states, an interaction (Davydov coupling) occurs leading to an exchange between the two identical excited parts of the dimer of alpha-phase 2-pyridone. It is interesting to note that the Davydov coupling parameters used in the present work are similar to those used by Maréchal and Witkowski in their pioneering work dealing with the adipic acid [21]. However, we shall introduce in the Davydov coupling some further flexibility by assuming it to be dependent on the $\mathrm{H}$-bond Bridge coordinate.

The complexity of the task to the experimental line shape of the cyclic dimer of alpha-phase 2-pyridone and to reproduce the corresponding isotope effect is determined by several factors, acting simultaneously. To take them into account, one needs to know a great number of parameters. For this idea, we think that the small residual discrepancies between theory and experiment are related to the neglect of some of these parameters: 1) assumption of a linear dependence of the angular frequency of the fast mode on the coordinate of the H-bond bridge [32], 2) assumption of the independence of the equilibrium position of the fast mode on the coordinate of the H-bond bridge [32], 3) assumption of a constant term for the Davydov coupling parameter [52], 4) neglect of electrical anharmonicity, 5) neglect of eventual weak tunneling through the potential barrier separating the two H-bond bridge minima, and 6) neglect of eventual relaxation mechanisms of non-adiabatic nature.

\section{Conclusions}

The presented theoretical approach is dealing within the strong anharmonic coupling theory according to which the high frequency mode and the H-bond bridge are anharmonically coupled through a linear dependence of the frequency of the fast mode on the elongation of the $\mathrm{H}$ bond bridge and takes into account Davydov coupling, Fermi resonances, anharmonicity of the H-bond Bridge and direct and indirect quantum relaxations. The present approach contains as special instances the majority precedent theoretical approach [53] dealing with the subject.

This approach, which is applied to reproduce the $v_{\mathrm{N}-\mathrm{H}(\mathrm{D})}$ IR spectra of centrosymmetric strongly bound dimers of alpha-phase 2-pyridone and their deuterated derivatives in the solid-state, is comprised in the linear response theory calculates the line shape by aid of the Fourier transform of the autocorrelation function of the dipole moment operator. The model has been applied to alphaphase 2-pyridone dimer crystal. It has been found that it is possible to correctly the experimental line shape of the hydrogenated compound and to predict satisfactorily the deuterium effect by using a set of spectral parameters. It appears from the present theoretical study that the simple model of Davydov coupling taking into account quantum indirect damping and the anharmonicity of the H-bond Bridge, is able to reproduce the experimental line shapes especially when the Fermi resonances were not ignored. 
In the end, we can say that the Fermi resonances appear play an important role to reproduce the experimental line shapes of alpha-phase 2-pyridone dimer crystal.

\section{Acknowledgements}

The authors thank Professor Henryk T Flakus from Institute of Chemistry, University of Silesia, 9 Szkolna Street, P1-40 006 Katowice, Poland for measuring the experimental spectra.

\section{REFERENCES}

[1] G. C. Pimentel and A. L. McClellan, "The Hydrogen Bond," W. H. Freeman, San Francisco, 1960.

[2] P. Schuster, G. Zundel and C. Sandorfy, "The Hydrogen Bond: Recent Developments in the Theory and Experiment, Parts I, II and III," Elsevier, Amsterdam, 1976.

[3] Y. Marechal, "The Hydrogen Bond and the Water Molecule," Elsevier, Amsterdam, Oxford, 2006.

[4] H. D. Arman, P. Poplaukhin and E. R. T. Tiekinkc, "2-Pyridone: Monoclinic Polymorph," Acta Crystallographica Section E: Structure Reports Online, Vol. 65, No. 12, 2009, p. 3187. doi:10.1107/S1600536809049496

[5] M. T. Boisdon, S. Castillo, J. F. Brazier, J. Favrot and C. J. Marsden, "2(1H)-Pyridinone(2-Pyridone): Self-Association and Association with Water Spectral and Structural Characteristics: Infrared Study and Ab Initio Calculations," Spectrochimica Acta Part A, Vol. 59, No. 14, 2003, pp. 3363-3377. doi:10.1016/S1386-1425(03)00162-8

[6] M. T. Boisdon, S. Castillo, J. F. Brazier and J. Favrot, "Vibrational Study of $2(1 \mathrm{H})$-Pyridinone(2-pyridone) in $\mathrm{H}_{2} \mathrm{O}$ and 1-D-2(1H)-Pyridinone(2-pyridone ND) in $\mathrm{D}_{2} \mathrm{O}$," Spectrochimica Acta Part A, Vol. 55, No. 7-8, 1999, pp. 1379-1388. doi:10.1016/S1386-1425(98)00301-1

[7] H. Ratajczak and W. J. Orville-Thomas, "Molecular Interactions," John Wiley \& Sons Inc., New York, 1980.

[8] G. R. Desiraju and T. Steiner, "The Weak Hydrogen Bond in Structural Chemistry and Biology," Oxford University Press, Oxford, 1999.

[9] M. Gallant, M. T. P. Viet and J. D. Wuest, "HydrogenBonded Dimers. Direct Study of the Interconversion of Pyridone Dimers and Hydroxypyridine Monomers by Low-Temperature Nuclear Magnetic Resonance Spectroscopy," Journal of the American Chemical Society, Vol. 113, No. 2, 1991, pp. 721-723. doi:10.1021/ja00002a077

[10] C. Fuke, T. Arao, Y. Morinaga, H. Takaesu, K. Ameno and T. Miyazaki, "Analysis of Paraquat, Diquat and Two Diquat Metabolites in Biological Materials by High-Performance Liquid Chromatography," Legal Medicine, Vol. 4, No. 3, 2002, pp. 156-163. doi:10.1016/S1344-6223(02)00011-1

[11] M. El-Kemary, J. A. Organero and A. Douhal, "Assessment of Solvent Effect on the Relaxation Dynamics of Milrinone, "Journal of Photochemistry and Photobiology A: Chemistry, Vol. 187, No. 2-3, 2007, pp. 339-347. doi:10.1016/j.jphotochem.2006.10.032

[12] Y. Ducharme and J. D. Wuest, "Use of Hydrogen Bonds to Control Molecular Aggregation. Extensive, Self-Complementary Arrays of Donors and Acceptors," Journal of Organic Chemistry, Vol. 53, No. 24, 1988, pp. 5787-5789. doi:10.1021/jo00259a037

[13] M. Gallant, M. T. P. Viet and J. D. Wuest, "Use of Hydrogen Bonds to Control Molecular Aggregation. Association of Dipyridones Joined by Flexible Spacers," Journal of Organic Chemistry, Vol. 56, No. 7, 1991 pp. 2284 2286. doi:10.1021/jo00007a007

[14] M. J. Wójcik, W. Tatara, M. Boczar, A. Apola and S. Ikeda, "Spectroscopic and Theoretical Study of Vibrational Spectra of Hydrogen-Bonded 2-Pyridone," Journal of Molecular Structure, Vol. 596, No. 1-3, 2001, pp. $207-$ 214. doi:10.1016/S0022-2860(01)00719-0

[15] M. J. Wójcik, "Theoretical Modeling of Vibrational Spectra and Multidimensional Proton Tunneling in Hydrogen-Bonded Systems," Journal of Molecular Liquids, Vol. 141, No. 1-2, 2008, pp. 39-46. doi:10.1016/j.molliq.2008.03.002

[16] M. J. Wójcik, "Theory of the Infrared Spectra of the Hydrogen Bond in Molecular Crystals," International Journal of Quantum Chemistry, Vol. 10, No. 5, 1976, pp. 747760. doi:10.1002/qua.560100506

[17] A. Witkowski and M. J. Wójcik, "Infrared Spectra of Hydrogen Bond a General Theoretical Model," Chemical Physics, Vol. 1, No. 1, 1973, pp. 9-16. doi:10.1016/0301-0104(73)87017-X

[18] M. J. Wójcik, "Fermi Resonance in Dimers: A Model Study," Molecular Physics, Vol. 36, No. 6, 1978, pp. 17571767. doi: $10.1080 / 00268977800102741$

[19] P. M. Morse, "Diatomic Molecules According to the Wave Mechanics. II. Vibrational Levels," Physics Reviews, Vol. 34 , No. 1,1929 , pp. 57-64. doi:10.1103/PhysRev.34.57

[20] N. Issaoui, N. Rekik, B. Oujia and M. J. Wójcik, "Theoretical Infrared Line Shapes of H-Bonds within the Strong Anharmonic Coupling Theory and Fermi Resonances Effects," International Journal of Quantum Chemistry, Vol. 110, No. 14, 2010, pp. 2583-2602. doi:10.1002/qua.22395

[21] Y. Maréchal and A. Witkowski, "Infrared Spectra of HBonded Systems," Journal of Chemical Physics, Vol. 48, No. 8, 1968, pp. 3697-3704. doi:10.1063/1.1669673

[22] N. Rösh and M. Ratner, "Model for the Effects of a Condensed Phase on the Infrared Spectra of HydrogenBonded Systems," Journal of Chemical Physics, Vol. 61, No. 8, 1974, pp. 3344-3351. doi:10.1063/1.1682497

[23] B. Boulil, O. H. Rousseau and P. Blaise, "Infrared Spectra of Hydrogen Bonded Species in Solution," Chemical Physics, Vol. 126, No. 2-3, 1988, pp. 263-290. doi:10.1016/0301-0104(88)85038-9

[24] H. T. Flakus, A. Tyl and A. Maślankiewicz, "ElectronInduced Phase Transition in Hydrogen-Bonded SolidState 2-Pyridone," Journal of Physical Chemistry A, Vol. 115, No. 6, 2011, pp. 1027-1039. doi:10.1021/jp108717v

[25] P. Blaise, M. J. Wójcik and O. H. Rousseau, "Theoretical Interpretation of the Line shape of the Gaseous Acetic Acid Dimer," Journal of Chemical Physics, Vol. 122, No. 
6, 2005, pp. 64306-64317. doi:10.1063/1.1847491

[26] M. J. Frisch, "Gaussian 03, Revision B.03," Gaussian, Inc., Pittsburgh, 2003.

[27] R. Kubo, "Statistical-Mechanical Theory of Irreversible Processes. I. General Theory and Simple Applications to Magnetic and Conduction Problems," Journal of Physics Society Japan, Vol. 12, No. 6, 1957, pp. 570-586. doi:10.1143/JPSJ.12.570

[28] R. Kubo, "Lectures in Theoretical Physics I," In: W. E. Brittin and L. G. DUnham, Eds., Interscience, 1st Annual Summer Institute for Theoretical Physics, Colorado, 16 June-22 August 1958, pp. 120-203.

[29] M. El-A. Benmalti, D. Chamma, P. Blaise and O. H. Rousseau, "Theoretical Interpretation of the Infrared Lineshape of Gaseous Propynoic and Acrylic Acid Dimers," Journal of Molecular Structure, Vol. 785, No. 1-3, 2006, pp. 27-31. doi:10.1016/j.molstruc.2005.09.036

[30] K. Heyne, N. Huse, J. Dreyer, E. T. J. Nibbering, T. Elsaesser and S. Mukamel, "Coherent Low-Frequency Motions of Hydrogen Bonded Acetic Acid Dimers in the Liquid Phase," Journal of Chemical Physics, Vol. 121, No. 2, 2004, pp. 902-913. doi:10.1063/1.1762873

[31] Z. Mielke and L. Sobczyk, "Vibrational Isotope Effects in Hydrogen Bonds," In: A. Kohen and H.-H. Limbach, Eds., Isotope Effects in Chemistry and Biology, CRC Press, Taylor and Francis Group, West Palm Beach, London, 2006, pp. 281-304.

[32] N. Issaoui, N. Rekik, B. Oujia and M. J. Wójcik, "Anharmonic Effects on Theoretical IR Line Shapes of Medium Strong H(D)Bonds," International Journal of Quantum Chemistry, Vol. 109, No. 3, 2009, pp. 483-499. doi:10.1002/qua.21839

[33] A. Novak, "Hydrogen Bonding in Solids Correlation of Spectroscopic and Crystallographic Data," Structure and bonding, Vol. 18, 1974, pp. 177-216.

[34] I. Olovsson and P. G. Jönsson, "Hydrogen Bond," In: P. Scheuster, G. Zundel and C. Sandorfy, Eds., Elsevier, Amsterdam, 1976, pp. 393-456.

[35] N. Rekik, B. Oujia and M. J. Wójcik, "Theoretical Infrared Spectral Density of H-bonds in Liquid and Gas Phases: Anharmonicities and Dampings Effect," Chemical Physics, Vol. 352, No. 1-3, 2008, pp. 65-76. doi:10.1016/j.chemphys.2008.05.009

[36] S. E. Odinokov and A. V. Iogansen, "Torsional $\gamma(\mathrm{OH})$ Vibrations, Fermi Resonance $[2 \gamma(\mathrm{OH}) v(\mathrm{OH})]$ and Isotopic Effects in i.r. Spectra of H-Complexes of Carboxylic Acids with Strong Bases," Spectrochimica Acta Part A: Molecular Spectroscopy, Vol. 28, No. 12, 1972, pp. 2343-2350. doi:10.1016/0584-8539(72)80214-9

[37] P. Blaise, M. J. Wójcik and O. H. Rousseau, "Theoretical Interpretation of the Line Shape of the Gaseous Acetic Acid Cyclic Dimer," Journal of Chemical Physics, Vol. 122, No. 6, 2005, p. 064306. doi:10.1063/1.1847491

[38] M. El-A. Benmalti, P. Blaise, H. T. Flakus and O. H. Rousseau, "Theoretical Interpretation of the Infrared Line Shape of Liquid and Gaseous Acetic Acid," Chemical Physics, Vol. 320, No. 2-3, 2006, pp. 267-274. doi:10.1016/j.chemphys.2005.07.032

[39] J. L. Leviel and Y. Marechal, "Infrared Spectra of HBonded Systems: Anharmonicity oh the H-Bond Vibrations in Cyclic Dimers," The Journal of Chemical Physics, Vol. 54 No. 3, 1971, pp. 1104-1107. doi:10.1063/1.1674943

[40] D. Hadzi, "Infrared Spectra of Strongly Hydrogen-Bonded Systems," Pure and Applied Chemistry, Vol. 11, No. 3, 1965, pp. 435-454. doi:10.1351/pac196511030435

[41] J. Emsley, "Very Strong Hydrogen Bonding," Chemical Society Reviews, Vol. 9, No. 1, 1980, pp. 91-124. doi:10.1039/cs9800900091

[42] W. H. Louisell and L. R. Walker, "Density-Operator Theory of Harmonic Oscillator Relaxation," Physical Review, Vol. 137, No. 1B, 1965, pp. B204-B211. doi:10.1103/PhysRev.137.B204

[43] R. Feynmann and F. Vernon, "The Theory of a General Quantum System Interacting with a Linear Dissipative System," Annals of Physics, Vol. 24, 1963, pp.118-173. doi:10.1016/0003-4916(63)90068-X

[44] W. H. Louisell, "Quantum Statistical Properties of Radiations," Wiley, New York, 1973.

[45] K. Belhayara, D. Chamma, A. Velcescu and O. H. Rousseau, "On the Similarity of the IR Lineshapes of Weak H-Bonds in the Gas and Liquid Phases: Quantum Combined Effects of Strong an Harmonic Coupling, Multiple Fermi Resonances, Weak Dampings and Rotational Structure," Journal of Molecular Structure, Vol. 833, No. 1-3, 2007, pp. 65-73. doi:10.1016/i.molstruc.2006.09.001

[46] L. C. Thomas, R. A. Chittenden and H. E. Hartley, "Hydrogen Bonding in Alkyl Phosphonic and Alkyl Phosphonothionic Acids," Nature, Vol. 192, 1961, pp. 1283-1284. doi:10.1038/1921283a0

[47] K. G. Tokhadze, G. S. Denisov, M. Wierzejewska and M. Drozd, "First Example of the ABC $v(\mathrm{OH})$ Absorption Structure for Both Gaseous and Crystalline Phase: Infrared Studies of Dimethylphosphinic Acid," Journal of Molecular Structure, Vol. 404, No. 1-2, 1997, pp. 55-62. doi:10.1016/S0022-2860(96)09360-X

[48] R. E Asfin, G. S. Denisov and K. G. Tokhadze, "The Infrared Spectra and Enthalpies of Strongly Bound Dimers of Phosphinic Acids in the Gas Phase. $\left(\mathrm{CH}_{2} \mathrm{Cl}\right)_{2} \mathrm{POOH}$ and $\left(\mathrm{C}_{6} \mathrm{H}_{5}\right)_{2} \mathrm{POOH}$," Journal of Molecular Structure, Vol. 608, No. 2-3, 2002, pp. 161-168. doi:10.1016/S0022-2860(01)00925-5

[49] H. T. Flakus, "On the Vibrational Transition Selection Rules for the Centrosymmetric Hydrogen-Bonded Dimeric Systems," Journal of Molecular Structure: Theochem, Vol. 187, 1989, pp. 35-53.

[50] H. T. Flakus and A. Miros, "Infrared Spectra of the Hydrogen Bonded Glutaric Acid Crystals: Polarization and Temperature Effects," Journal of Molecular Structure, Vol. 484, No. 1-3, 1999, pp. 103-115.

[51] H. T. Flakus and M. Jabonska, "Study of Hydrogen Bond Polarized IR Spectra of Cinnamic Acid Crystals," Journal of Molecular Structure, Vol. 707, No. 1-3, 2004, pp. $97-$ 108.

[52] D. Chamma and O. H. Rousseau, "Infrared Spectra of 
weak H-Bonds: Beyond an Adiabatic Description of Fermi Resonances," Chemical Physics, Vol. 248, No. 1, 1999 , pp. 91-104.

[53] N. Issaoui, H. Ghalla and B. Oujia, "Theoretical Study of
Hydrogen and Deuterium Bond in Glutaric Acid Crystal Dimer," International Journal of Quantum Chemistry, Vol. 112, No. 4, 2012, pp. 1006-1015. 